\title{
A new fourth-order four stage explicit trigonometrically-fitted Runge-Kutta-Nyström method for solving periodic problems
}

\begin{abstract}
In this work, a new fourth order four stage explicit trigonometrically-fitted Runge-KuttaNyström (ETFRKN) method for the numerical solution of second order initial value problems with periodic solutions based on Simos technique is constructed. The numerical results show the efficiency of the new method in comparison with other existing methods.
\end{abstract}

Keyword: Trigonometric fitting; Rungeï Kuttaï Nyström method; Periodic problems 